\title{
Changes in photosynthesis and chlorophyll fluorescence in two soybean (Glycine max) varieties under $\mathrm{NaCl}$ stress
}

\author{
Bin Luo ${ }^{1,2}$, Cheng Wang ${ }^{1,2}$, Xiaodong Wang ${ }^{1,2}$, Han Zhang ${ }^{1,2}$, Yanan Zhou ${ }^{1,2}$, \\ Wensen Wang ${ }^{1,3 *}$, Peng Song ${ }^{4 *}$ \\ (1. Beijing Research Center for Information Technology in Agriculture, Beijing Academy of Agriculture and Forestry Sciences, \\ Beijing 100097, China; \\ 2. National Engineering Research Center for Information Technology in Agriculture, Beijing 100097, China; \\ 3. State Grid Shenyang Electric Power Supply Company, Shenyang 110866, China; \\ 4. College of Plant Science and Technology, Huazhong Agricultural University, Wuhan 430074, China)
}

\begin{abstract}
Traditional detection methods of crop information are often destructive and low efficiency. In this study, a new evaluation method was developed based on photosynthesis and chlorophyll fluorescence. Via analysis of the changes under $\mathrm{NaCl}$ stress during the seedling stage of two varieties, the salt resistance mechanism of soybeans was explored and a non-destructive stress-recognition method was developed. In this experiment, two soybean varieties were treated with one of four levels of $\mathrm{NaCl}$ stress: CK (0 mmol/L), LS (50 mmol/L), MS (100 mmol/L), and HS (150 mmol/L), for $15 \mathrm{~d}$. The normal functions of the photosynthetic system of soybeans were enhanced under $\mathrm{LS} \mathrm{NaCl}$ stress, but were inhibited under $\mathrm{HS} \mathrm{NaCl}$ stress. Biomass, net leaf photosynthetic rate $(\mathrm{Pn})$, stomatal conductance $\left(g_{s}\right)$, intercellular carbon dioxide concentration $(\mathrm{C} i)$, transpiration rate $(T r)$, chlorophyll fluorescence parameters Y(II) and PSII decreased. However, in contrast to the findings of other studies on the influence of severe drought stress on soybean for long periods in which non-photochemical quenching coefficient $(q N)$ decreased, this parameter increased under salt stress in soybean. The results demonstrate that the method developed is a promising tool for rapid and non-destructive detection of soybean photosynthetic responses under salt stress in the field.
\end{abstract}

Keywords: biomass, chlorophyll fluorescence parameters, $\mathrm{NaCl}$ stress, photosynthetic parameters, soybean DOI: $10.25165 /$ j.ijabe.20211403.5941

Citation: Luo B, Wang C, Wang X D, Zhang H, Zhou Y N, Wang W S, et al. Changes in photosynthesis and chlorophyll fluorescence in two soybean (Glycine max) varieties under $\mathrm{NaCl}$ stress. Int J Agric \& Biol Eng, 2021; 14(3): 76-82.

\section{Introduction}

Soybean (Glycine max) is a major source of high-quality protein and oil for human consumption ${ }^{[1]}$. Soybean production may be limited by environmental stress factors, such as soil salinity $^{[2]}$. It is well known that salt stress can cause marked inhibition of photosynthetic efficiency and restrict crop survival rate, yield, while degrading the quality of agricultural products ${ }^{[3-6]}$.

In recent years, soil salinization has become a growing global trend ${ }^{[7,8]}$, especially in Asia and Africa, thereby posing a serious threat to crop yields and ecological environments in these regions ${ }^{[9,10]}$. Therefore, improvement and utilization of soil

Received date: 2020-06-03 Accepted date: 2021-03-08

Biographies: Bin Luo, $\mathrm{PhD}$, research interests: crop information detection, Email: luob@nercita.org.cn; Cheng Wang, PhD, Professor, research interests: agricultural intelligent equipment, Email: wangc@nercita.org.cn; Xiaodong Wang, PhD, research interests: plant physiology, Email: wangxd@nercita.org.cn; Han Zhang, Master, research interests: crop information detection, Email: zhangha@nercita.org.cn; Yanan Zhou, Master, research interests: sensor technology, zhouyn@ nercita.org.cn.

*Corresponding author: Wensen Wang, PhD, Assistant Professor, research interests: sensor technology. State Grid Shenyang Electric Power Supply Company Dadong Electric Power Supply Company, 66th Jinqiao Road, Shenyang 111000, China. Tel: +86-15084009595, Email: 346443247@qq.com; Peng Song, PhD, Associate Professor, research interest: crop phenotyping, agricultural robot, Huazhong Agricultural University, No. 1 Shizishan street, Hongshan District, Wuhan 430070, Hubei, China. Tel: +86-18515360082, Email: songp@mail.hzau.edu.cn. salinity can effectively relieve problems such as low grain productivity, crop species restrictions, and ecosystem damage caused by world population growth and the concomitant reduction of arable land ${ }^{[11]}$. China is subject to a considerable land salinization problem and a high growth rate of land salinization. The area of saline land is currently about 100 million $\mathrm{hm}^{2[12]}$. Soil salinization has occurred in major soybean-producing areas of China at different degrees of severity. Since ancient times, soybeans have been an important grain and oil crop, as well as an industrial raw material rich in protein and unsaturated fatty acids in $\mathrm{China}^{[13]}$. Soybean is a moderately salt-tolerant crop, and $\mathrm{NaCl}$ stress will exert a certain influence on the survival of soybean plants and soybean yield ${ }^{[14]}$. Therefore, an understanding of how to select salt-tolerant soybean varieties and how to predict salt stress can facilitate increases in soybean yield effectively in saline and alkaline areas.

Preceding reports have laid a certain research foundation on the influence of salt stress on plants-research carried out by Liu et al. ${ }^{[15]}$ indicated that salt stress would influence plant root growth and restrict biomass growth. Research implemented by Cayuela et al. ${ }^{[16]}$ showed that $\mathrm{NaCl}$ stress could cause physiological alterations in seeds. The findings of Porgali and Yurekli ${ }^{[17]}$ proved that salt stress would degrade leaf enzymatic activity. However, most studies have used biochemical indicators, spectral information, biomass information, and photosynthetic parameters, whereas few studies have used chlorophyll fluorescence kinetics to analyze the influence of salt stress on plants. Chlorophyll 
fluorescence kinetics refers to the technical means to analyze light energy distribution information of plant leaves through chlorophyll fluorescence and makes rapid, non-destructive, and accurate detection and analysis of photosynthetic organs and even the health status of the plant itself ${ }^{[18-20]}$.

Therefore, soybean seedlings of salt-tolerant and salt-sensitive cultivars were grown in potted medium culture under four salinity-stress levels. Calculation and analysis of trends and differential relationships of biomass information, photosynthetic indicators, and fluorescence parameters were conducted to provide a data base foundation for salt stress and plant photosynthesis. The salt-tolerant mechanism of salt-tolerant cultivars was analyzed. Finally, a drought versus salt stress identification method was proposed.

\section{Materials and methods}

\subsection{Experimental materials and processing}

This experiment was implemented in Xiaotangshan National Precision Agricultural Research and Demonstration Base $\left(40^{\circ} 18^{\prime} \mathrm{N}\right.$, $\left.116^{\circ} 45^{\prime} \mathrm{E}\right)$ in December 2016. Sunshine duration was from 8:00 am to 5:00 pm, the maximum temperature in daytime was $30^{\circ} \mathrm{C}$, and the minimum temperature at night was $18^{\circ} \mathrm{C}$. The soil type used in the experiment was ordinary seedling medium, which contains nutrients as follows: organic matter content at $8.76 \%$, total nitrogen content at $0.31 \%$, available potassium content at $110 \mathrm{mg} / \mathrm{kg}$, available phosphorus content at $34.1 \mathrm{mg} / \mathrm{kg}, \mathrm{pH} 7.4$, and maximum moisture content at $80 \%$. Plastic pots with $11 \mathrm{~cm}$ inner diameters, $25 \mathrm{~cm}$ height, and holes in the bottom were used; the dry weight of the soil in each pot was $2 \mathrm{~kg}$. Soybean cultivars used in the experiment were salt-sensitive "Qihuang 35" and salt-tolerant "Xudou 14" provided by Shandong Shofine Seedling Technology Co., Ltd (Figure 1).

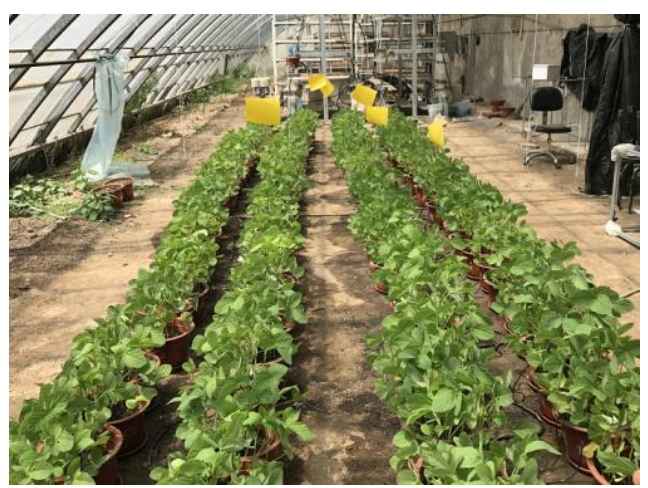

Figure 1 Potted experiment samples of soybean

The experiment was carried out in a greenhouse. Seedlings were sown in five holes in each pot (two seedlings in each hole), at a depth of $2.5 \mathrm{~cm}$. After $15 \mathrm{~d}$, three healthy soybean seedlings in each pot with uniform growth were selected for culture under $\mathrm{NaCl}$ stress treatment and under non-saline control conditions. The four $\mathrm{NaCl}$ levels of stress applied include: CK $(0 \mathrm{mmol} / \mathrm{L})$, LS $(50 \mathrm{mmol} / \mathrm{L})$, MS (100 mmol/L), and HS $(150 \mathrm{mmol} / \mathrm{L})^{[21]}$. Water of the above salinities was used to wash seedlings five times on the first day of stress treatment. To prevent salt content loss and any salinity changes, trays were placed at the bottom of these pots, water was replenished every day, to maintain maximum moisture content at $80 \%$, and water of each corresponding concentration was used for washing every $5 \mathrm{~d}$. The first set of data was obtained on day 0 after stress; subsequently, data acquisition was conducted every $5 \mathrm{~d}$, stress treatment lasted $15 \mathrm{~d}$, and a total of four groups of data were collected.

\subsection{Measuring items and methods}

\subsubsection{Biomass measurement}

On the 16th day of salt stress treatment at the seedling stage, water was used to repeatedly wash the soil in the pots, and roots were completely drawn out of the pots and rapidly brought to the lab in a cooler. Tap water was used to wash the shoot part and underground parts, and the moisture was wiped to dry off the plant body surface. Four complete plants of each variety under each stress treatment were selected to measure fresh weights of shoot part. Afterward, samples were de-enzymized by steaming at $105^{\circ} \mathrm{C}$ for $40 \mathrm{~min}$ and then dried to constant weight at $75^{\circ} \mathrm{C}$ for $12 \mathrm{~h}$. Then, root dry weights of the shoot parts were measured. A scale was used to measure seedling biomass.

Root to shoot ratio = Root dry weight/Shoot part dry weight

Salt sensitivity index $=($ Plant dry weight under stress

- Dry weight of control plant)

/Plant dry weight under stress $\times 100^{[22]}$

\subsubsection{Measurement of gas exchange parameters}

The Li-6400 Portable Photosynthesis System (LICOR Inc., USA) was used to determine gas exchange parameters starting from 9:00 am to 10:00 am on each measurement day ${ }^{[23]}$. Four pots were included in each group under stress treatment; one leaf of the second layer was randomly selected from each pot for measurement. Measurement values of gas exchange parameters were recorded five times every $2 \mathrm{~s}$. The measurement was repeated 20 times. The concentration of carbon dioxide used in the measurement was $0.69 \mathrm{mg} / \mathrm{L}$, light intensity was $1000 \mu \mathrm{mol} /\left(\mathrm{m}^{2} \cdot \mathrm{s}\right)$, leaf chamber temperature was set to $22^{\circ} \mathrm{C}$, and measurement area was $6 \mathrm{~cm}^{2}$. Determination parameters included: net photosynthetic rate of leaf $(P n)$, stomatal conductance $\left(g_{s}\right)$, intercellular carbon dioxide concentration $(\mathrm{Ci})$, and transpiration rate $(T r)$.

2.2.3 Measurement and acquisition of chlorophyll fluorescence parameters

Self-developed fluorescence detection equipment was adopted, the excitation light source is blue LED array, power is more than $180 \mathrm{~W}$, fluorescence imaging resolution is superior $640 \times 480$, the sampling rate is greater than 12 frames/s. Determination on each measurement day started from 8:00 pm to 12:00 am (dark adaptation duration was more than $2 \mathrm{~h}$ ), leaves at the second layer of soybean seedlings were picked to gauge, which was repeated five times. The measurement mode is that after sufficient dark adaptation, minimum fluorescence $F o$ was recorded. Saturated fluorescence of $400 \mu \mathrm{mol} /\left(\mathrm{m}^{2} \cdot \mathrm{s}\right)$ was used to excite and record maximum fluorescence $\mathrm{Fm}$ in dark adaptation. Actinic light of $800 \mu \mathrm{mol} /\left(\mathrm{m}^{2} \cdot \mathrm{s}\right)$ was used, saturated flash was applied every $5 \mathrm{~s}$, after pulse measurement was performed 15 times, and maximum fluorescence (averaging the last three times) $\mathrm{Fm}^{\prime}$ and steady-state fluorescence $F s$ under light adaptation were recorded. Actinic light was closed, and minimum fluorescence $F o^{\prime}$ was recorded under light adaptation.

Fluorescence parameters were calculated according to the following equations:

PSII transformation efficiency of primary light energy,

$$
F v / F m=(F m-F o) / F m
$$

PSII Actual photochemical quantum yield,

$$
Y(\mathrm{II})=\Phi \mathrm{PSII}=\left(F m^{\prime}-F s\right) / F m^{\prime}
$$

Photochemical quenching coefficient,

$$
q P=1-\left(F s-F o^{\prime}\right) /\left(F m^{\prime}-F o^{\prime}\right)
$$

Non-photochemical quenching coefficient,

$$
q N=1-\left(F m^{\prime}-F o^{\prime}\right) /(F m-F o)
$$


where, $F v$ is the variable fluorescence; PS(II) is Photosystem II.

2.2.4 Data arrangement and analysis

Microsoft Excel 2016 was used to conduct statistical data analysis; Origin 9.1 was used to make function plots, and Microsoft Word 2007 was used to elaborate the tables.

\section{Results}

\subsection{Effects of salt stress of soybean on biomass}

As shown in Table 1, the seedling-stage biomass of the two soybean cultivars under $50 \mathrm{mmol} / \mathrm{L}$ (LS) stress exceeded those under $0 \mathrm{mmol} / \mathrm{L}$ (CK). However, as stress level increased, biomass decreased. Under $150 \mathrm{mmol} / \mathrm{L}$ (HS) salt stress, total biomass reached 90.5\% (Qihuang 35) and 89.1\% (Xudou 14) of CK values. Although the total biomass of the two cultivars showed an increasing trend under salt stress, their energy and biomass distribution modes were entirely different. There was a significant increase in the root:shoot ratio of Qihuang 35, and after salinity reached $177.0 \%$ of $\mathrm{CK}$ under $100 \mathrm{mmol} / \mathrm{L}$ (MS) $\mathrm{NaCl}$ stress, it declined to $139.2 \%$ under HS stress. In contrast, root:shoot ratio of Xudou 14 basically maintained an insignificant difference with $\mathrm{CK}$ under salt stress with $\mathrm{NaCl}$ at different concentrations $(p \leq 0.05)$.

Table 1 Biomass of Qihuang 35 and Xudou 14 after salt stress $(p \leq 0.05)$

\begin{tabular}{|c|c|c|c|c|c|c|c|c|}
\hline Varieties & Treat & Stalk fresh weight & Root fresh weight & Total fresh weight & Stalk dry weight & Root dry weight & Total dry weight & Root to shoot ratio \\
\hline \multirow{3}{*}{ Qihuang35 } & $\mathrm{CK}$ & $1.175^{\mathrm{b}}$ & $2.825^{\mathrm{b}}$ & $4.000^{\mathrm{c}}$ & $0.299^{\mathrm{b}}$ & $0.076^{\mathrm{b}}$ & $0.376^{\mathrm{b}}$ & $0.255^{\mathrm{c}}$ \\
\hline & LS & $2.250^{\mathrm{a}}$ & $4.025^{\mathrm{a}}$ & $6.275^{\mathrm{a}}$ & $0.466^{\mathrm{a}}$ & $0.129^{\mathrm{a}}$ & $0.596^{\mathrm{a}}$ & $0.279^{c}$ \\
\hline & HS & $1.950^{\mathrm{a}}$ & $2.425^{\mathrm{b}}$ & $4.375^{\mathrm{b}}$ & $0.254^{\mathrm{c}}$ & $0.087^{\mathrm{b}}$ & $0.341^{\mathrm{c}}$ & $0.356^{\mathrm{b}}$ \\
\hline \multirow{4}{*}{ Xudou14 } & $\mathrm{CK}$ & $2.475^{\mathrm{a}}$ & $4.400^{\mathrm{a}}$ & $6.875^{\mathrm{a}}$ & $0.422^{b}$ & $0.178^{b}$ & $0.600^{\mathrm{b}}$ & $0.427^{\mathrm{a}}$ \\
\hline & LS & $2.875^{\mathrm{a}}$ & $4.100^{\mathrm{b}}$ & $6.975^{\mathrm{a}}$ & $0.48675^{\mathrm{a}}$ & $0.221^{\mathrm{a}}$ & $0.708^{\mathrm{a}}$ & $0.453^{\mathrm{a}}$ \\
\hline & MS & $2.450^{\mathrm{a}}$ & $3.525^{\mathrm{c}}$ & $5.975^{\mathrm{b}}$ & $0.374^{\mathrm{c}}$ & $0.165^{\mathrm{bc}}$ & $0.539^{\mathrm{c}}$ & $0.445^{\mathrm{a}}$ \\
\hline & HS & $2.025^{\mathrm{b}}$ & $3.075^{\mathrm{d}}$ & $5.100^{\mathrm{c}}$ & $0.37875^{\mathrm{c}}$ & $0.156^{\mathrm{c}}$ & $0.535^{\mathrm{c}}$ & $0.418^{\mathrm{a}}$ \\
\hline
\end{tabular}

Note: Different lowercase letters indicate significant differences among treatments.

As shown in Table 2, sensitivities of biomass of both cultivars under LS stress increased to some extent. However, this sensitivity declined with the increase of salt stress. The degree of salt sensitivity of the underground part of Qihuang 35 was under low concentration and gradually decreased under MS and HS treatments, but this was higher than $\mathrm{CK}$ as salt concentration increased. Differences in sensitivities between MS and HS treatments and $\mathrm{CK}$ of the underground part and the shoot part of Xudou 14 were basically minor, and sensitivity of the root and the stalk of Xudou 14 basically kept within $15 \mathrm{~d}$ under MS and HS treatments. The yield of soybean is defined by the shoot part; salt sensitivity in this passage is based on the shoot part salt sensitivity index.

Table 2 Salt sensitivity index of Qihuang 35 and Xudou 14 after salt stress

\begin{tabular}{lcccc}
\hline Varieties & Treat & $\begin{array}{c}\text { Stalk salt } \\
\text { sensitivity index }\end{array}$ & $\begin{array}{c}\text { Root salt } \\
\text { sensitivity index }\end{array}$ & $\begin{array}{c}\text { Total salt } \\
\text { sensitivity index }\end{array}$ \\
\hline \multirow{2}{*}{ Qihuang 35 } & LS & 35.71046 & 40.84778 & 36.82886 \\
& MS & -12.0561 & 37.09016 & 3.337612 \\
& HS & -18.0118 & 11.80695 & -10.4025 \\
\hline \multirow{2}{*}{ Xudou 14 } & LS & 13.30252 & 19.43503 & 15.21893 \\
& MS & -12.8342 & -7.54148 & -11.2089 \\
& HS & -11.4191 & -13.8978 & -12.1439 \\
\hline
\end{tabular}

3.2 Effects of salt stress on the soybean gas exchange parameters

Seedling-stage soybeans under $\mathrm{NaCl}$ stress and changes of $P n$ of different cultivars under different levels of $\mathrm{NaCl}$ stress were inconsistent. Under $100 \mathrm{mmol} / \mathrm{L}$ and $150 \mathrm{mmol} / \mathrm{L}(\mathrm{HS})$ treatments, $P n$ of Qihuang 35 decreased with the increase of $\mathrm{NaCl}$ stress since the 5th day under stress. However, Pn of Xudou 14 was higher than that of CK under MS and HS stress treatments since the 10th day. Under HS treatment, $P n$ values of Qihuang 35 and Xudou 14 were lower than that in $\mathrm{CK}$ since the 5th day, and since the 10th day, $P n$ values of Qihuang 35 were significantly lower than that of CK plants. Under the 15-day HS treatment, $P n$ of salt-sensitive Qihuang 35 decreased by $47.3 \%$, whereas that of salt-tolerant species, Xudou 14, decreased by $6.9 \%$, only. Thus, $P n$ of Qihuang 35 seedlings was much more significantly affected by $\mathrm{NaCl}$ stress than that of Xudou 14 seedlings (Figure 2a).

Under LS treatment, $g_{s}$ of Qihuang 35 and Xudou 14 were basically consistent with CK, namely, both initially increased and then declined with time. However, with the increase of $\mathrm{NaCl}$ stress (MS and HS), $g_{s}$ of the two cultivars significantly decreased with the increase of stress magnitude. Moreover, after the 5th day from stress treatment initiation, $g_{s}$ of seedlings of the two cultivars under study changed relatively steadily under HS treatment. $g_{s}$ on the 5 th day was reduced by $76.4 \%$ and $72.3 \%$ when compared with CK; however, $g_{s}$ only decreased by $77.3 \%$ and $53.4 \%$ on the 15 th day (Figure 2b).

Under MS and HS treatments, concentration trend of intercellular carbon dioxide of the two species was basically consistent with the trends in $P n$ described above. However, under HS treatment, $C i$ of Qihuang 35 seedlings on the 15th day after stress was slightly higher than that on the 5th day, whereas intracellular $\mathrm{Ci}$ change trend of Xudou 14 was basically consistent with $P n$ change rate (Figure 2c). The influence of $\mathrm{NaCl}$ stress on $\operatorname{Tr}$ of seedlings of the two cultivars was basically consistent with the effects described for $g_{s}$ (Figure 2d).

\subsection{Effects of salt stress on soybean chlorophyll fluorescence parameters}

Transformation efficiencies of PSII primary light energy $(F v / F m)$ in the two cultivars under $100 \mathrm{mmol} / \mathrm{L}$ and $150 \mathrm{mmol} / \mathrm{L}$ (HS), were insignificantly lower than for CKs on the 5th day and maintained a consistent trend thereafter. Under $150 \mathrm{mmol} / \mathrm{L}$ treatment, $F v / F m$ of non-salt-tolerant Qihuang 35 was insignificantly lower than for CKs on the 5th day, abruptly declined after, was significantly higher than for CKs on the 10th day, and significantly decreased below CK levels on the 15th day. Salt-tolerant species, Xudou 14, started being either significantly or insignificantly higher than for CKs on the 5th day, it slowly decreased after the 10th day and was insignificantly lower than in CKs on the 15th day (Figure 3a). PSII actual photochemical quantum yields $Y$ (II) of basically all soybean seedlings were significantly 
lower than for CKs under LS, MS, and HS treatments, and their increase amplitudes were magnified with increasing stress magnitude and respectively reached 27.9\% (Qihuang 35) and 23.6\% (Xudou 14 ) of CK on the 15th day under HS treatment (Figure 3b).

$q P$ of Qihuang 35 increased with increasing stress in the initial stage (i.e., 5th day); however, with time, $q P$ s under MS and HS treatments on the 5th day increased compared with CKs, whereas $q P$ under HS treatment was significantly lower than under CK and MS treatments. $q P$ of Xudou 14 seedlings was higher than that under CK treatment with increasing stress $5 \mathrm{~d}$ before stress

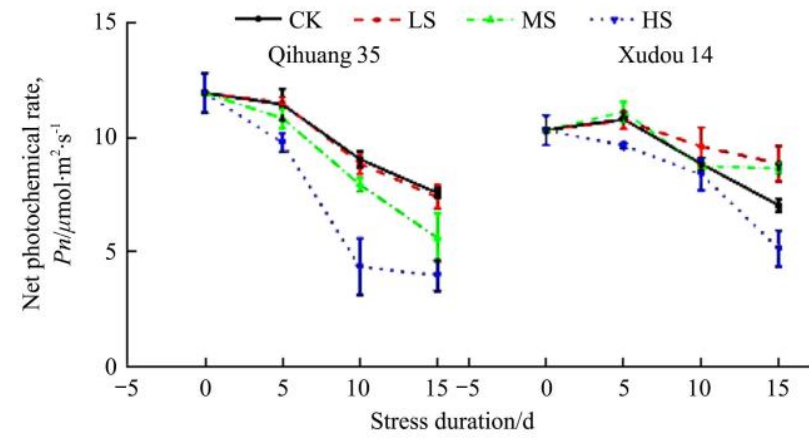

a.

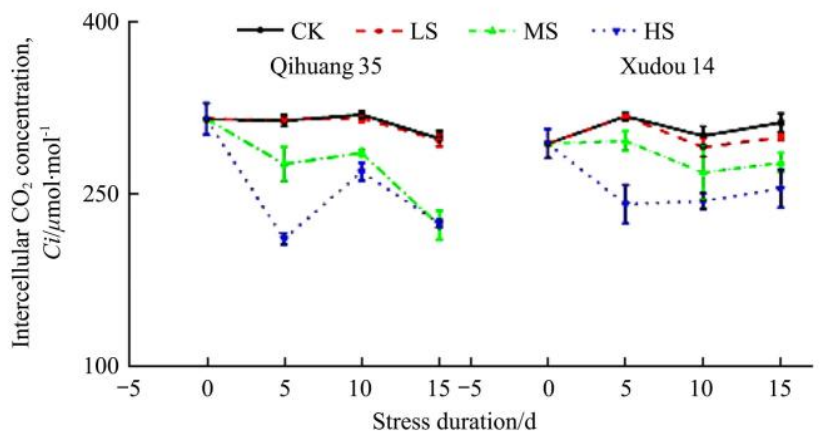

c.

Figure 2 Effects of salt stress on $\mathrm{Pn}, \mathrm{g}_{s}, \mathrm{Ci}$, and $\mathrm{Tr}$ treatment and after the 10th day. The difference was smaller than for CKs as stress level remained unchanged (Figure 3c). $q N$ of seedlings of the two species increased with increasing stress level (HS $>$ MS $>$ LS). 14th day after stress treatment, $q N$ of Qihuang 35 under HS treatment was lower than that under LS and MS treatments. However, $q N$ of Xudou 14 remained higher than that under LS and MS treatments (Figure 3d).

From the chlorophyll fluorescence imaging of two soybean varieties (Figure 4), the chlorophyll fluorescence parameters were significantly different under different treatments.

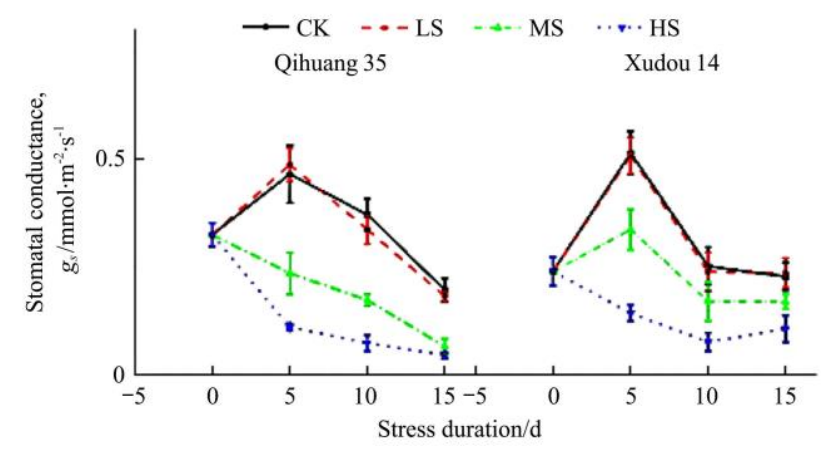

b.

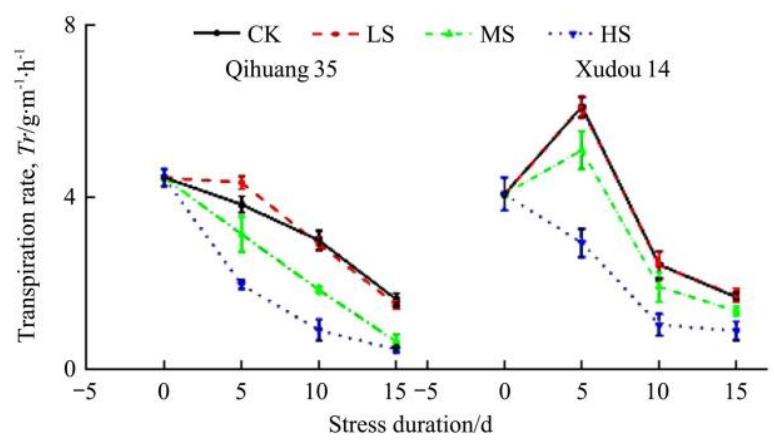

d.

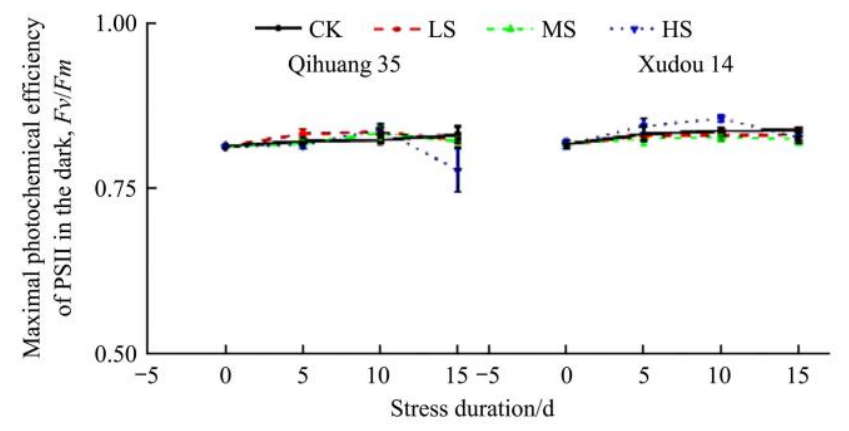

a.

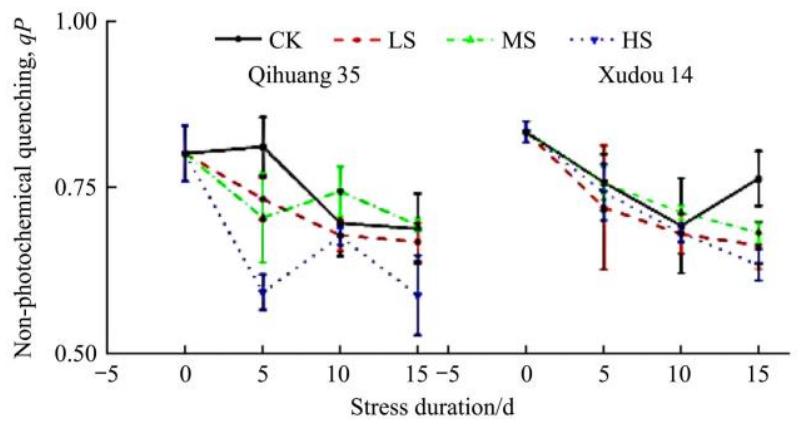

c.

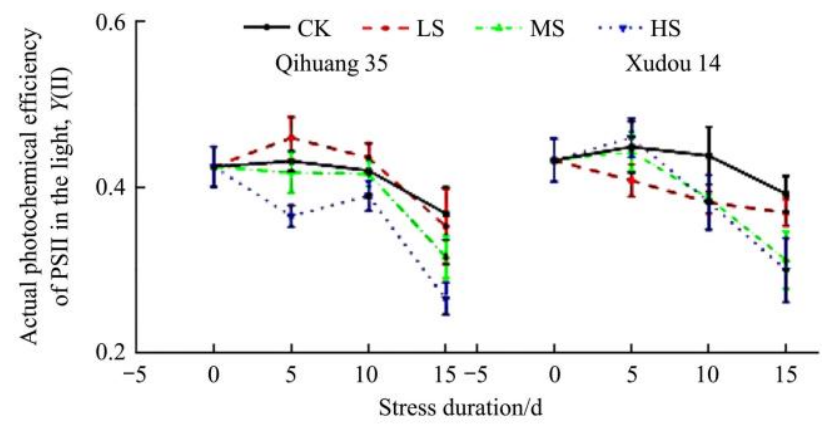

b.

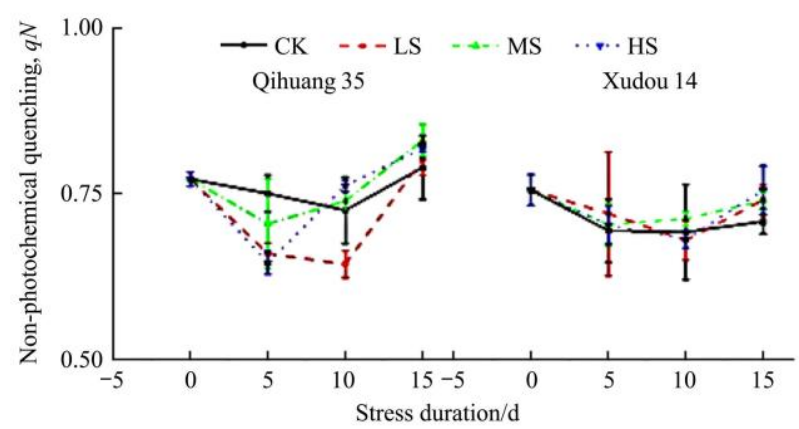

d.

Figure 3 Effects of salt stress on $F v / F m, Y($ II $), q P$ and $q N$ 


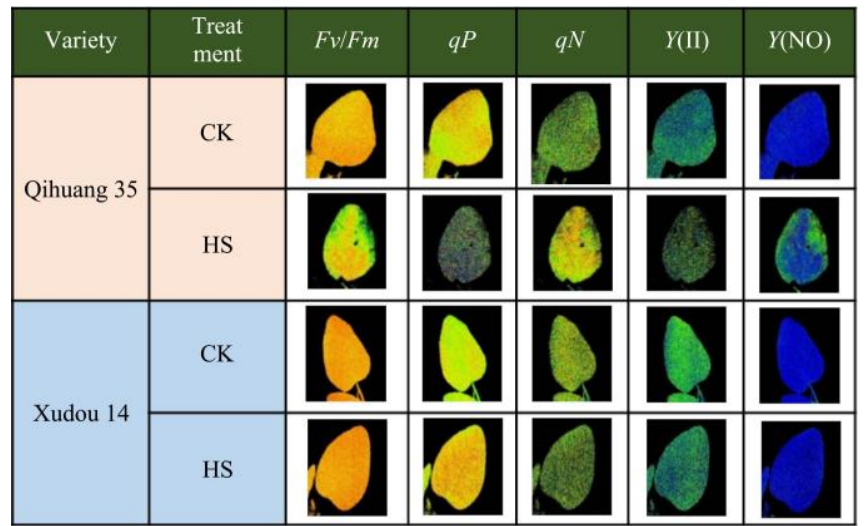

Figure 4 Chlorophyll fluorescence imaging of two soybean varieties

\section{Discussion}

Both biomass change and partitioning are the important features of plant stress-tolerant performance ${ }^{[24]}$. Results of many experiments have indicated that a certain concentration of $\mathrm{NaCl}$ can boost the increase of plant biomass ${ }^{[25-27]}$. In this experiment, a low-concentration of $\mathrm{Na}^{+}$and $\mathrm{Cl}^{-}$ions $(50-100 \mathrm{mmol} / \mathrm{L})$ promoted biomass growth in the two soybean varieties at seedling stage. The biomass of salt-sensitive Qihuang 35 was increased by $58.3 \%$, and the biomass of salt-tolerant Xudou 14 was increased by $17 \%$. However, as salinity increased, biomass decreased. The biomass of both cultivars under high $\mathrm{NaCl}$ concentration stress reached $90 \%$ of the control samples. The two cultivars had different salt resistance mechanisms. To guarantee normal growth of the shoot parts, the salt-sensitive species allocated more biomass or energy to the main organ and the roots under salt stress. Under high salt stress, moisture could not be normally absorbed even with the distribution of an increasing amount of biomass, and biomass of the shoot part only reached $84.7 \%$. This compensation effect in the non-sensitive cultivar Xudou 14, under low salt concentration was lower than that of the sensitive cultivar Qihuang 35; the root:shoot ratio kept within 40\%-45\%, and stalk biomass was lower than that at $150 \mathrm{mmol} / \mathrm{L}(89.7 \%$ of the CK). Root:shoot ratio was the corresponding strategy used by plants under stress to partition biomass so as to guarantee growth of the shoot part. Moreover, the root salt-sensitive index of Qihuang 35 increased sharply at low $\mathrm{NaCl}$ concentration, but it sharply decreased under high $\mathrm{NaCl}$ concentration. Conversely, the underground salt sensitive index of Xudou 14 was stable across treatments. Therefore, Qihuang 35 prevented the damage of salt stress by controlling root growth. It has been also established that "reduced root growth under salinity stress is an adaptive mechanism of plant to reduce its root surface area to prevent excess sodium absorption/uptake". However, the root could not fully develop, and the total biomass was low under high salt stress. Thus, plant salt tolerance might be contrary to its sensitivity degree to salt. Local soil saline-alkali concentration should be taken into consideration to obtain maximum crop yield for the growth of agricultural crops in saline-alkali soil.

$P n$ of plants is one of the important indicators of plant photosynthesis status. $\mathrm{Pn}, \mathrm{g}_{s}, \mathrm{Ci}$, and $\mathrm{Tr}$ of soybean seedlings under high $\mathrm{NaCl}$ concentration stress decreased. However, under low salt stress, the extent of reduction in $P n$ in NaCl-tolerant Xudou 14 was smaller than in non-NaCl-tolerant Qihuang 35. Under $100 \mathrm{mmol} / \mathrm{L} \mathrm{NaCl}$, indicators of Xudou 14 only kept insignificant differences relative to $\mathrm{CK}$, but indicators of Qihuang
35 under this stress started being significantly lower than CK since the 5th day. Moreover, under $\mathrm{NaCl}$ stress, factors limiting $P n$ were different and were divided into stomatal and non-stomatal limitations ${ }^{[28]}$. The former refers to the mechanism which regulates stomatal size to reduce $\mathrm{CO}_{2}$ diffusion into the mesophyll, thus limiting $P n$ to avoid damage caused to photosynthetic organs. The latter refers to the mechanism regulating the effect of the photosynthetic system that could not yet completely evade damage caused by excessive photosynthesis to plant organs which will then lose partial photosynthetic capacity, whereby $P n$ decreased. The method proposed by Farquhar and Sharkey ${ }^{[29]}$ is commonly used to detect the reason for photosynthesis rate decline, namely, the trends in $g_{s}$ and $\mathrm{Ci}$ changes are monitored; if $g_{s}$ and $\mathrm{Ci}$ simultaneously decrease or rise when $P n$ decreases, then the reason for the decline of photosynthesis rate is a stomatal limitation. On the other hand, if $g_{s}$ and $\mathrm{Ci}$ do not correlate linearly or if they correlate negatively, then the reason for a photosynthesis rate decline is likely a non-stomatal limitation. Under $\mathrm{NaCl}$ treatment, $g_{s}$ and $C i$ trends in the two cultivars remained basically consistent. Therefore, the general reason for the decline of photosynthesis rates of seedlings of the two cultivars under medium and low-NaCl-concentration stress was a stomatal limitation, while the photosynthetic system did not suffer any damage from $\mathrm{NaCl}$ stress. However, under high-NaCl-concentration treatment, change trends of $g_{s}$ and $\mathrm{Ci}$ in non-NaCl-tolerant Qihuang 35 were contrary to previous results since the 5th day, but $g_{s}$ and $C i$ of NaCl-tolerant species did not change; thus, the main reason for the decline of $P n$ of Qihuang 35 was a non-stomatal limitation, whereas the reason for the decline in $P n$ of Xudou 14 under any stress level was the same in all cases, namely, a non-stomatal limitation.

In recent years, chlorophyll fluorescence kinetics has been applied and developed in the plant detection field because of its advantages such as non-destructive, rapid, and accurate ${ }^{[30,31]}$. Chlorophyll fluorescence kinetics analyzes and reflects light energy transport and distribution information by analyzing light energy absorption by plant photosynthetic organs and the photosynthetic system, namely, chlorophyll fluorescence, photosynthetic electron transport, and heat consumption. $F v / F m$ and transformation efficiency of plant primary light energies reflect health conditions of the PSII system. In plants under stress, $F v / F m$ will initially rise and then decline by relying on the compensation effect of the plant itself with increasing stress over time. In this experiment, under high-salt-concentration stress, $F v / F m$ initially increased and then decreased. $\quad F v / F m$ of the salt-tolerant cultivar experienced the compensation effect only under high-salt-concentration stress. However, the non-salt-tolerant cultivar experienced the compensation effect even under low-salt-concentration stress, which was basically consistent with its salt sensitivity indicator. Thus, it can be speculated that $F v / F m$ sensitivity was consistent with salt sensitivity, as the compensation effect appearing under lower-salt-concentration stress makes this cultivar more sensitive to salt. However, under high-salt-concentration stress, the compensation effect was much smaller than the damage caused by stress on the photosynthetic system of soybean seedlings and then abruptly decreased from significantly higher than $\mathrm{CK}$ to significantly lower than CK. Y(II) is the actual photosynthesis rate of PSII. In this experiment, Y(II)s of both cultivars tested under medium and high-NaCl-concentration stress decreased; salt stress would degrade actual photosynthetic capacity of soybean seedlings and influence dry matter accumulation of soybean plants. 
These results are consistent with reports of the effects of heavy-metal stress ${ }^{[32]}$ and drought stress ${ }^{[33]}$ on soybean plants.

Additionally, $q P$ and $q N$ represent transformation efficiency of light energy and heat consumption capacity of plants, respectively, and the sum of the two is inversely proportional to the damage caused to plants by light energy under stress ${ }^{[34]}$. Under medium and low salt-concentration stress, $q P$ and $q N$ of both soybean cultivars increased as salt stress increased. However, under high salt concentration, $q P$ and $q N$ of Qihuang 35 were basically lower than that under $\mathrm{CK}$ and those under other stress conditions. In contrast, $q P$ and $\mathrm{qN}$ of Xudou 14 were basically higher than that under $\mathrm{CK}$ and other stress conditions. Under medium and low-salt-concentration stress, plant compensation effect was higher than the obstruction degree of electron transport rate of the photosynthetic system. Under severe salt stress, electron transport rate of photosynthetic system in non-salt-tolerant species was obstructed, thereby causing $q P$ to decline to a large extent.

Stress caused by soil abnormalities is the main reason for ecological imbalance and reduced crop yield. Recognition and timely treatment of soil stress can improve ecological environment of crops. A large number of research results have shown that drought stress for a certain period caused photosynthetic electron transport rate and heat consumption capacity of soybean plants to decrease $(q P \text { and } q N \text {, respectively })^{[35,36]}$. However, in this experiment, as stress time increased under medium- and low-salt-concentration conditions, heat consumption capacity of soybean plants increased. Thus, using chlorophyll fluorescence parameters to judge whether the stress borne by plants is drought stress or salt stress is feasible. The results in this study can provide a new theoretical foundation for preventing and discriminating between drought and salt stress.

\section{Conclusions}

1) High-NaCl-concentration stress inhibited dry matter accumulation of soybean seedlings, especially dry matter accumulation in the shoot part. Change of distribution strategies of dry matter and energy of the salt-sensitive cultivar (Qihuang 35) was more significant than that of the salt-tolerant cultivar (Xudou 14); more biomass was partitioned to the plant part experiencing the stress, namely, the roots.

2) Under $50 \mathrm{mmol} / \mathrm{L}$ or $100 \mathrm{mmol} / \mathrm{L} \mathrm{NaCl}$ stress, dry matter accumulation of the two cultivars was affected. Hence, the main functions of the photosynthetic system were under a stomatal limitation, and as salt concentration increased, photosynthetic system or organs of the salt-sensitive species were damaged easily. The main factor restricting the photosynthetic system was changed into a non-stomatal limitation. Under $150 \mathrm{mmol} / \mathrm{L} \mathrm{NaCl}$ stress, the main factor affecting photosynthesis of the salt-tolerant cultivar was a stomatal limitation, whereas that affecting the salt-sensitive cultivar was a non-stomatal limitation.

3) Under high-NaCl-concentration stress, transformation efficiency of PSII primary light energy and actual photochemical quantum yield of soybean seedlings significantly decreased. $q P$ and $q N$ of the two cultivars increased under $50 \mathrm{mmol} / \mathrm{L}$ and $100 \mathrm{mmol} / \mathrm{L}$ salt treatments increasing level of stress. $q P$ and $q N$ of Xudou 14 increased with increasing stress even under $150 \mathrm{mmol} / \mathrm{L} \mathrm{NaCl}$. However, the opposite results were obtained for Qihuang 35. Evaluation of salt tolerance of the two cultivars can be carried out by judging critical values of $q P$ and $q N$ with increased $\mathrm{NaCl}$ stress. Drought and salt stress of soybeans can be differentiated through changes of $q P$ and $q N$.
4) The results demonstrate that the method is a promising tool for rapid and non-destructive diagnosis of soybean under salt stress in the field.

\section{Acknowledgements}

This work was financially supported by the Key-Area Research and Development Program of Guangdong Province (Grant No. 2019B020214005), Science and Technology Innovation Special Construction Funded Program of Beijing Academy of Agriculture and Forestry Sciences (Grant No. KJCX20170418), the National Natural Science Foundation of China (Grant No. 21974012)

\section{[References]}

[1] Yu Y, Hou W S, Yael H, Sun S, Wu C X, Ifat M, et al. Constitutive expression of feedback-insensitive cystathionine Y-synthase increases methionine levels in soybean leaves and seeds. Journal of Integrative Agriculture, 2018; 17(1): 54-62

[2] Ghassemi-Golezani K, Taifeh-Noori M, Oustan S, Moghaddam M. Response of soybean cultivars to salinity stress. Journal of Food Agriculture \& Environment, 2009; 7(2): 401-404

[3] Arjun K, Kevin G, Vincent P, Jaya Y, Alex S, Heike B. Beneficial plant microbe interactions and their effect on nutrient uptake, yield, and stress resistance of soybeans. In: Soybean-biomass, yield and productivity, 2018; pp. 1-20.

[4] Feng Z, Ding C Q, Li W H, Wang D C, Cui D. Applications of metabolomics in the research of soybean plant under abiotic stress. Food Chemistry, 2020, 310: 125914. doi: 10.1016/j.foodchem.2019.125914.

[5] Tang X L, Mu X M, Shao H B, Wang H Y, Brestic M. Global plant-responding mechanisms to salt stress: physiological and molecular levels and implications in biotechnology. Critical Reviews in Biotechnology, 2015; 35(4): 425-437.

[6] Hou Q Z, Sun K, Zhang H, Su X, Fan B Q, Feng H Q. The responses of photosystem II and intracellular ATP production of Arabidopsis leaves to salt stress are affected by extracellular ATP. Journal of Plant Research, 2018; 131: 331-339.

[7] Turan M A, Elkarim A H A, Taban N, Taban S. Effect of salt stress on growth, stomatal resistance, proline and chlorophyll concentrations on maize plant. African Journal of Agricultural Research, 2009; 4(9): 893-897.

[8] Chang X M, Gao Z Y, Wang S L, Chen H R. Modelling long-term soil salinity dynamics using SaltMod in Hetao Irrigation District, China. Computers and Electronics in Agriculture, 2019; 156: 447-458.

[9] Wang J L, Huang X J, Zhong T Y, Chen Z G. Review on sustainable utilization of salt-affected land. Acta Geographica Sinica, 2011; 66(5): 673-684. (in Chinese)

[10] Bimpong I K, Manneh B, Sock M, Diaw F, Amoah N K A, Ismail A M, et al. Improving salt tolerance of lowland rice cultivar 'Rassi' through marker-aided backcross breeding in West Africa. Plant Science, 2016; 242: 288-299.

[11] Huang L B, Bai J H, Chen B, Zhang K J, Huang C, Liu P P. Two-decade wetland cultivation and its effects on soil properties in salt marshes in the Yellow River Delta, China. Ecological Informatics, 2012; 10: 49-55.

[12] Liu L, Bai X G, Jiang Z D. The generic technology identification of saline-alkali land management and improvement based on social network analysis. Cluster Computing, 2019; 22(6): 13167-13176.

[13] Chen J N, Tang G Y, Zhou J F, Liu W, Bi Y L. The characterization of soybean germ oil and the antioxidative activity of its phytosterols. RSC Advances, 2019; 9(68): 40109-40117.

[14] Khan M S A, Chowdhury J A, Razzaque M, Ali M, Paul S, Aziz M. Dry matter production and seed yield of soybean as affected by post-flowering salinity and water stress. Bangladesh Agronomy Journal, 2016; 19(2): 21-27.

[15] Liu W, Li R J, Han T T, Cai W, Fu Z W, Lu Y T. Salt stress reduces root meristem size by nitric oxide-mediated modulation of auxin accumulation and signaling in Arabidopsis. Plant Physiology, 2015; 168(1): 343-356.

[16] Cayuela E, Pérez-Alfocea F, López D M C, Bolarín M C. Priming of seeds with $\mathrm{NaCl}$ induces physiological changes in tomato plants grown under salt stress. Physiologia Plantarum, 2010; 96(2): 231-236.

[17] Porgali Z B, Yurekli F. Salt stress-induced alterations in proline 
accumulation, relative water content and superoxide dismutase (SOD) activity in salt sensitive Lycopersicon esculentum and salt-tolerant $L$. pennellii. Acta Botanica Hungarica, 2005; 47(1): 173-182.

[18] Maxwell K, Johnson G N. Chlorophyll fluorescence-a practical guide. Journal of Experimental Botany, 2000; 51(345): 659-668.

[19] Dhara A, Satish C B. Melatonin and nitric oxide relulate sunflower deedling growth under salt stress accompanying differential expression of $\mathrm{Cu} / \mathrm{Zn}$ SOD and Mn SOD. Free Radical Biology and Medicine, 2017; 106: 315-328.

[20] Maxim Y G, Paul G F. Using chlorophyll fluorescence kinetics to determine photosynthesis in aquatic ecosystems. Limnology and oceanography, 2020; 66: 1-13.

[21] Hairmansis A, Berger B, Tester M, Roy S J. Image-based phenotyping for non-destructive screening of different salinity tolerance traits in rice. Rice, 2014; 7(16): 1-10.

[22] Rejili M, Vadel A M, Guetet A, Neffatti M. Effect of $\mathrm{NaCl}$ on the growth and the ionic balance $\mathrm{K}^{+} / \mathrm{Na}^{+}$of two populations of Lotus creticus (L.) (Papilionaceae). South African Journal of Botany, 2007; 73(4): 623-631.

[23] Wang W S, Wang C, Pan D Y, Zhang Y K, Luo B, Ji J W. Effects of drought stress on photosynthesis and chlorophyll fluorescence images of soybean (Glycine max) seedlings. Int J Agric \& Biol Eng, 2018; 11(2): 196-201

[24] Poorter H, Niklas K J, Reich P B, Oleksyn J, Poot P, Mommer L. Biomass allocation to leaves, stems and roots: meta-analyses of interspecific variation and environmental control. New Phytologist, 2012; 193(1): $30-50$.

[25] Galli V, da Silva Messias R, Perin E C, Borowski J M, Bamberg A L, Rombaldi C V. Mild salt stress improves strawberry fruit quality. LWT, 2016; 73: 693-699.

[26] He X, Yang P L, Ren S M, Li Y K. Quantitative response of oil sunflower yield to evapotranspiration and soil salinity with saline water irrigation. Int J Agric \& Biol Eng, 2016; 9(2): 63-73.

[27] Ventura Y, Myrzabayeva M, Alikulov Z, Omarov R, Khozin-Goldberg I, Sagi M, et al. Effects of salinity on flowering, morphology, biomass accumulation and leaf metabolites in an edible halophyte. AoB Plants, 2014; 6(20): 55-64.

[28] Sarabi B, Fresneau C, Ghaden N, Bolandnazar S, Streb P, Badeck F W, et al Stomatal and non-stomatal limitations are responsible in down-regulation of photosynthesis in melon plants grown under the saline condition: Application of carbon isotope discrimination as a reliable proxy. Plant Physiology and Biochemistry, 2019; 141: 1-19.

[29] Farquhar G D, Sharkey T D. Stomatal conductance and photosynthesis. Annual Review of Plant Physiology, 1982; 33(1): 74-79.

[30] Lichtenthaler H K, Buschmann C, Rinderle U, Schmuck G. Application of chlorophyll fluorescence in ecophysiology. Radiation \& Environmental Biophysics, 1986; 25(4): 297-308.

[31] Sun J, Zhou X, Mao H P, Wu X H, Zhang X D, Gao H Y. Identification of pesticide residue level in lettuce based on hyperspectra and chlorophyll fluorescence spectra. Int J Agric \& Biol Eng, 2016; 9(6): 231-239.

[32] Hu H Q, Wang L H, Wang Q Q, Jiao L Y, Hua W Q, Zhou Q, et al Photosynthesis, chlorophyll fluorescence characteristics, and chlorophyll content of soybean seedlings under combined stress of bisphenol A and cadmium. Environmental Toxicology and Chemistry, 2015; 33(11): 2455-2462.

[33] Prieto P, Pecluelas J, Llusia J, Asensio D, Marc E. Effects of long-term experimental night-time warming and drought on photosynthesis, $F \mathrm{~V} / F \mathrm{~m}$ and stomatal conductance in the dominant species of a Mediterranean shrubland. Acta Physiologiae Plantarum, 2009; 31(4): 729-739.

[34] Yang S P, Wei C Z, Liang Y C. Effects of $\mathrm{NaCl}$ stress on the characteristics of photosynthesis and chlorophyll fluorescence at seedlings stage in different sea island cotton genotypes. Scientia Agricultura Sinica, 2010; 43(8): 1585-1593. (in Chinese)

[35] Sun H F, Zhan Y, Wei L J, Liu S L, Zhang H B, Lin H R, et al. Effects of drought on chlorophyll fluorescence parameters in flowering of soybean. Agricultural Research in the Arid Areas, 2008; 26(2): 61-64. (in Chinese)

[36] Sheng J G, Li X M, Gu K L, Hu D F. Effect of drought stress on photosynthetic characteristics of soybean leaves during flowering period. Guizhou Agricultural Sciences, 2015; 43(11): 52-54. (in Chinese) 